\title{
Utilidad del tratamiento con oxcarbacepina en pacientes con diagnóstico de trastorno por abuso/dependencia de cocaína
}

\author{
Juan José llopis llácer*; Agurtzane Castillo Aguilella** \\ *Psiquiatra. UCA Dep. Salud 02. Generalitat Valenciana. Profesor Asociado, Departamento de Psicobiología Universitat Jaume I Castelló. \\ * Psicóloga. Departamento de Toxicomanías CRE. Castelló.
}

Enviar correspondencia a:

Juan José Llopis jllopis@psb.uji.es

\section{RESUMEN}

Evaluamos la eficacia de la Oxcarbacepina en el tratamiento de la adicción a cocaína, y su influencia sobre la impulsividad y el craving en cocainómanos.

Metodología. Estudio clínico observacional prospectivo de pacientes diagnosticados de abuso/dependencia de cocaína (criterios DSM IV) en tratamiento con Oxcarbacepina en Seguimiento de 12 semanas.

Muestra. 30 pacientes mayores de edad que manifestaron voluntad de tratar en una Unidad de Conductas Adictivas (UCA). La selección se realizó al azar previa petición de autorización y consentimiento informado.

Resultados. A lo largo del seguimiento la tasa de abstinencia va aumentando hasta alcanzar al $100 \%$ de la muestra que se mantiene en tratamiento a los 3 meses $160 \%$ de los que iniciaron tratamiento). Progresivamente van aumentando los resultados en urinoanálisis negativos hasta alcanzar un 90,9\% en la semana 8, de los que están en tratamiento.

En nuestro estudio, a pesar de las limitaciones relativas al tamaño de la muestra, y el porcentaje (40\%) de abandonos al inicio del tratamiento, encontramos que mediante el tratamiento con Oxcarbacepina se producen descensos estadísticamente significativos del craving y la impulsividad a las 4, 8 y 12 semanas, respecto de la medida basal al inicio del tratamiento. La disminución del craving presenta el correlato conductual del descenso en el consumo.

Conclusiones. La oxcarbacepina se muestra eficaz en la reducción de la impulsividad y también sobre el craving de cocaína.

Recomendamos dosis iniciales elevadas en aquellos de pacientes con mayor impulsividad al inicio de tratamiento.

Palabras clave: cocaína, dependencia cocaína, craving, impulsividad, Oxcarbacepina, tratamiento farmacológico.

\section{ABSTRACT}

To assess the efficacy of oxcarbazepine in the treatment of cocaine addiction and its influence on impulsivity and craving in cocaine addicts.

Methodology. A prospective, observational and clinical study was conducted in patients diagnosed with cocaine abuse/ dependence (DSM IV criteria), monitoring supervised treatment with oxcarbazepine for 12 weeks.

Sample. 30 patients (aged 18 or over) voluntarily undergoing treatment at an Addictive Behaviors Unit (UCA). Patients were selected randomly after providing explicit authorization and informed consent.

Results. Abstinence rate increased, reaching $100 \%$ of participants that remained in compliance throughout the 12 -week period (60\% of those who started the treatment). Negative results in the urine test for cocaine detection also increased, reaching $90.9 \%$ of those in treatment at week 8 .

Treatment with oxcarbazepine was seen to produce a statistically significant reduction in craving and impulsivity after 4,8 and 12 weeks, with respect to baseline measures at the start of treatment. The craving index correlates with reduction in cocaine use.

Conclusions. Oxcarbazepine is effective in reducing impulsivity and craving in relation to cocaine.

Thus, we recommend high initial doses of oxcarbazepine in patients with high impulsivity prior to treatment.

Key words: cocaine, dependence, craving, impulsivity, oxcarbazepine, pharmacological treatment. 


\section{INTRODUCCIÓN}

E los últimos años la evolución del consumo y de las tendencias en los movimientos del mercado de estupefacientes ha convertido a España en el cuarto país del mundo donde más cocaína se incauta, después de Colombia, Estados Unidos y Venezuela, y el primero de Europa. Si las cifras de aprehensiones son un reflejo del incremento de la eficacia policial en la represión del gran tráfico, también lo son de la demanda del mercado de ese país. Por tanto las cifras de consumo no van a ser menos escalofriantes: de los potenciales 13,3 millones de consumidores de cocaína en el mundo, según las estimaciones de las Naciones Unidas $^{1}$ el 2,6\% son españoles. Por primera vez nos encontramos por delante de americanos $(2,5 \%)$, irlandeses $(2,4 \%)$ e ingleses $(2,1 \%)$.

En la presentación de la Encuesta sobre Uso de Drogas en Enseñanzas Secundarias 2004 se destacó que el consumo de cocaína entre los adolescentes españoles de 14 a 18 años se había multiplicado por cuatro en el último decenio de estudio (1994-2004)². En el informe anual de 2004 del Observatorio Español sobre Drogas (OED), España era el país de la Unión Europea donde se registró un mayor número de cocainómanos que solicitaron tratamiento de deshabituación de esta droga. Concretamente de algo más del $20 \%$ de cocainómanos que accedían por primera vez en 1994 se pasó a cerca de un 40\% en el 2004 que iniciaron tratamiento en las redes asistenciales públicas ${ }^{3}$.

Desde hace ya unos años, los profesionales de la clínica vemos cómo la mayoría de las demandas de tratamiento por problemas relacionados con drogas tienen detrás la adicción a la cocaína. Adicción que ha desplazado a la de la heroína como "droga diana», término utilizado por los expertos para indicar la sustancia cuyo consumo, ya convertido en problemático, lleva al usuario a solicitar ayuda.

Como vemos, el consumo de cocaína está alcanzando tasas por encima de las peores previsiones y precisamente es en esta dirección que nuestros esfuerzos, como profesionales, se dirigen cada vez más a realizar un tratamiento integral con alternativas eficaces que incluyan terapia psicológica y farmacológica, y posibiliten un mejor abordaje del adicto y su patología desde el primer momento de la demanda, ya que muchas veces ese primer momento de tratamiento es el que ofrece mayores dificultades de contención. En este sentido, el tratamiento del paciente cocainómano está siendo objeto de un mayor esfuerzo por encontrar las terapias farmacológicas cada vez más adecuadas.

Diversos estudios vinculan la impulsividad con la adicción a cocaína, la frecuencia del consumo e intensidad del craving. Así mismo el consumo de alcohol parece estar íntimamente relacionado con el de cocaí- na4. Es así como se ha hipotetizado sobre la utilidad de fármacos antiepilépticos para reducir la impulsividad y el consumo de alcohol

Kile Kamppman ${ }^{5}$ realizó una revisión bibliográfica en la que mostraba como tanto las neuronas gabaérgicas como las glutamatérgicas parecían mostrarse importantes moduladores del sistema de recompensa. Por ello los fármacos que afectaban a la transmisión gabaérgica y glutamatérgica podían reducir las propiedades gratificantes de la cocaína y al deseo de consumo de esta sustancia. Utilizando topiramato (anticonvulsionante que eleva las concentraciones cerebrales de GABA, facilita la transmisión gabaérgica e inhibe la actividad glutamatérgica en los receptores de AMPA/kainato), Kamppman presenta resultados que muestran como el tratamiento aumenta la probabilidad de adherencia al tratamiento y abstinencia a la cocaína frente al tratamiento con placebo.

En esta línea de acción farmacológica la Gabapentina ${ }^{6}$ puede tener una función importante en el tratamiento de las adicciones incrementando el nivel de dopamina, noradrenalina y serotonina, lo que a efectos clínicos resulta beneficioso en el tratamiento de las adicciones. Principalmente eleva el GABA, siendo su mecanismo de acción algo complicado, pero de un amplio espectro. Su uso puede sustituir a fármacos como las benzodiacepinas dado que tiene muy pocas interacciones, produce menos trastornos cognitivos y efectos adversos. Así contribuye a que los pacientes estén tranquilos, relajados y sin trastornos de sueño.

Prieto $^{6}$ expone que los efectos de la administración de la Gabapentina en pacientes toxicómanos podría ser útil para manejar la dependencia y el craving por su acción sobre el sistema glutamatérgico y dopaminérgico. Al respecto de los mecanismos de acción del glutamato en la adicción a la cocaína se encuentra bibliografía que apunta en la misma dirección $n^{5,7}$

El craving se ha mostrado como uno de los factores más determinantes en la evolución de la adicción a cocaína ${ }^{8}$. Aunque no existe un acuerdo unánime, los autores creen que este podría ser el factor determinante en las recaídas en el consumo de cocaína, en cambio en lo que todos los autores parecen coincidir es en la necesidad de abordar terapéuticamente el craving así como la impulsividad que parece caracterizar a lo consumidores / adictos de cocaína en particular y de psicoestimulantes en general|5,8-11.

En el tratamiento de la dependencia y el manejo del craving en cocainómanos se han empleado antidepresivos, antipsicóticos y benzodiacepinas con los que se logra sedar al paciente pero no disminuir el craving ${ }^{12}$, por lo que la línea de actuación en los últimos años se dirige hacia el uso de anticonvulsionantes. Estos fármacos, por su perfil de acción, tendrían una mayor especificidad sobre el control de impulsos evitando en la medida de lo posible y dependiendo de 
sus características farmacodinámicas los efectos de sedación no deseados ${ }^{6}$. En estos estudios se muestra la posible utilidad de los antiepilépticos de acción sobre los mecanismos glutamatérgico de control de la impulsividad, como el Topiramato, la Gabapentina o la Oxcarbacepina ${ }^{6,12,13}$.

Las experiencias clínicas de uso de la Carbamacepina en el tratamiento del síndrome de abstinencia alcohólico y en el craving se han visto limitadas, en algunos casos, por los efectos secundarios del fármaco, sus propiedades farmacocinéticas y la necesidad de monitorización de niveles plasmáticos.

La introducción en el mercado de la Oxcarbacepina, antiepiléptico de acción antagonista del canal calcio, farmacológicamente emparentado con la carbamacepina, pero con la ventaja de un metabolito activo, la 10-hidroxicarbacepina, con similares capacidades terapéuticas pero sin las limitaciones de aquella para el uso clínico, permite aventurar la posibilidad de que sea un fármaco útil para el control de la impulsividad conductual, los trastornos del control de impulsos y en pacientes con abuso/dependencia de cocaína y también del abuso de alcohol con impulsividad asociada ${ }^{14,15}$.

Utilizando Oxcarbacepina a una dosis de 600 mgr./ día se han mostrado reducciones significativas en las puntuaciones medias de los pacientes en las escalas de Barratt y $\mathrm{CCO}$, sin que se produzcan efectos adversos relevantes ${ }^{11,13,14}$.

En el presente estudio pretendemos valorar la eficacia de la Oxcarbacepina en el tratamiento de la adicción a cocaína, sola o asociada a trastorno por abuso de alcohol y su influencia sobre la impulsividad y el craving en cocainómanos.

Existe abundante bibliografía acerca de la pérdida de control de impulsos entre los consumidores de cocaína y el alto nivel de craving que manifiestan al iniciar tratamiento ${ }^{5,8}$. Nuestra hipótesis de partida es que el uso de la Oxcarbacepina (en la dosis establecida como óptima al inicio del estudio: 600-900 mgr.) reduce el craving de cocaína y la impulsividad entre los pacientes en tratamiento.

\section{MÉTODOS}

El diseño utilizado es el de estudio clínico observacional prospectivo que evalúa la evolución de pacientes diagnosticados de abuso/dependencia de cocaína (criterios DSM IV) en tratamiento con Oxcarbacepina en un seguimiento durante 12 semanas.

Treinta pacientes mayores de edad que demandan tratamiento en la Unidad de Conductas Adictivas por presentar abuso/dependencia de cocaína. El tamaño de la muestra no es muy amplio lo que ya de por si puede representar limitaciones en el estudio.

La selección de los pacientes se realizó al azar previa petición de autorización y consentimiento informado, para su participación en el estudio en base a los siguientes criterios de inclusión: mayor de edad, diagnostico principal de abuso /dependencia a cocaína e intención de tratamiento voluntario. Los criterios de exclusión fueron: psicopatología aguda grave o descompensada, diagnostico de demencia, tratamiento con otros antiepilépticos, embarazo o tratamiento con anovulatorios, y antecedentes de hipersensibilidad a Carbamacepina.

Todos los pacientes recibieron un protocolo de tratamiento consistente en 500 mgr. diarios de CDP Colina durante 20 días y Oxcarbacepina en dosis crecientes desde 300 mgr. hasta conseguir la dosis establecida como optima para el estudio de 600-900 mgr./ día en unas dos semanas.

La tasa de rechazo a participar en el estudio fue muy baja ya que tan solo 4 pacientes que iniciaron tratamiento por adicción a cocaína, y cumplían los criterios de inclusión en el estudio, declinaron su participación.

Estudiamos diversas variables sociodemográficas y de consumo correlacionándolas con el craving de cocaína, medido mediante la escala $\mathrm{CCO}^{16,17}$, Escala Analógica Visual de Craving ${ }^{14,18}$ y los niveles de impulsividad registrados mediante la Escala de Impulsividad de Barratt $17,19,20$

Con el fin de validar nuestra hipótesis hemos utilizados dos tipos de datos:

1. Datos obtenidos mediante la utilización de escalas de medida para la evaluación del craving y la impulsividad (tabla 1).

Tabla 1. Medidas utilizadas en el estudio.

\begin{tabular}{|l|l|}
\hline MEDIDAS DE CRAVING & MEDIDAS DE IMPULSIVIDAD \\
\hline & - Impulsividad cognitiva de Barratt \\
- Escala Analógica Visual de & $\begin{array}{l}\text { - Impulsividad motora de Barratt } \\
\text { Craving (EAV) }\end{array}$ \\
- Impulsividad no planeada de \\
CCO de cocaína & Barratt \\
& - Impulsividad total de Barratt \\
\hline
\end{tabular}

2. Correlatos conductuales respecto al consumo de cocaína a lo largo del tratamiento. Se valora si han existido consumos de cocaína y alcohol en las diferentes fases de la realización del estudio, así como la frecuencia de los mismos y la cantidad consumida. 
3. En todos los casos se efectuaron analíticas de orina (benzoilecgonina) cada 3 días, para asegurar la veracidad de la información que aportaba el paciente.

Análisis estadístico. Para el análisis de las variables utilizamos el paquete estadístico SPSS de Windows, versión 11.0. Realizamos análisis descriptivo de frecuencias, prueba t de Student para las comparaciones entre variables y análisis de varianza (ANOVA), estableciendo un nivel de significación de $p<.05$.

\section{RESULTADOS}

\section{Consumo de cocaína}

La muestra inicial se compone de 30 pacientes con diagnostico de dependencia de cocaína, con edades comprendidas entre los 19 y 42 años y una edad media de 27 años. El consumo de cocaína se produce: vía pulmonar (10\%), mucosas $(80 \%)$ y mucosa combinada con pulmonar (10\%).

La historia de consumo de cocaína varía entre los 2 y 15 años, situándose la media en 7 años de consumo. La edad de inicio en el consumo varía entre los 15 y los 30 años, situándose la mediana de la muestra en 19 años. El 70.9 \% de la muestra ha realizado consumo de cocaína en el último mes. El consumo realizado para este grupo se localiza en los últimos 10 días previos al inicio del tratamiento (76,2\%). La cantidad consumida es variable pero la mayoría $(70,9 \%)$ de la muestra ha realizado un consumo en los últimos 30 días de entre 1y 10 gramos.

La abstinencia de Cocaína en los pacientes en tratamiento a lo largo del seguimiento aumenta desde el inicio y alcanza al $100 \%$ de la muestra que se mantiene en tratamiento al final de aquel (60\% de abstinencia de los que inician tratamiento).

El incremento en hallazgos de urinoanálisis negativos (figura 1) se observa de forma progresiva desde las primeras mediciones (pasando del 30\% inicial al $82 \%$ en la semana 4 para alcanzar hasta un $90,9 \%$ en la semana 8 y finalmente el 100 de abstinencia a los 3 meses), en los pacientes retenidos en tratamiento. En una primera analítica sistemática de sangre no se encuentran valores patológicos destacables para los parámetros analizados.

Con respecto al consumo del resto de sustancias. En la primera analítica de sangre no encontramos valores compatibles con dependencia alcohólica. Uno de los pacientes realizaba consumo de abuso de alcohol superior a 4 ube's semanales. El 73,3\% de la muestra ha sido consumidor de cannabis manteniéndose en el uso esporádico el $26,7 \%$ de ella y habitual en el $20 \%$ de los casos.
Hemos de destacar que el descenso porcentual en la abstinencia a cannabis y alcohol $(90.9 \%$ a los 2 meses y $85 \%$ a los tres meses), se produce por un artefacto estadístico como resultado del descenso del tamaño muestral, $40 \%$ de perdidas. En realidad aumenta el porcentaje relativo pero no el número de pacientes que realizan consumos.

\section{Evolución del craving}

En las mediciones del craving de cocaína, el tratamiento con Oxcarbacepina parece conseguir un descenso de las puntuaciones obtenidas a lo largo de las 12 semanas de estudio, tal y como se muestra en las Figuras 1 y 2. La disminución en los valores del craving presenta el correlato conductual del descenso en el consumo hasta alcanzar el $100 \%$ de abstinencia a las 12 semanas en los pacientes que continúan en tratamiento. El contraste $t$ de medias de muestras relacionadas, muestra un descenso estadísticamente significativo: escala analógica visual de craving: ( $p<$ 0.001): descenso de 4,25 puntos a los 3 meses de tratamiento; CCO ( $p<0.001$ ): descenso de 71,42 puntos en la puntuación final.

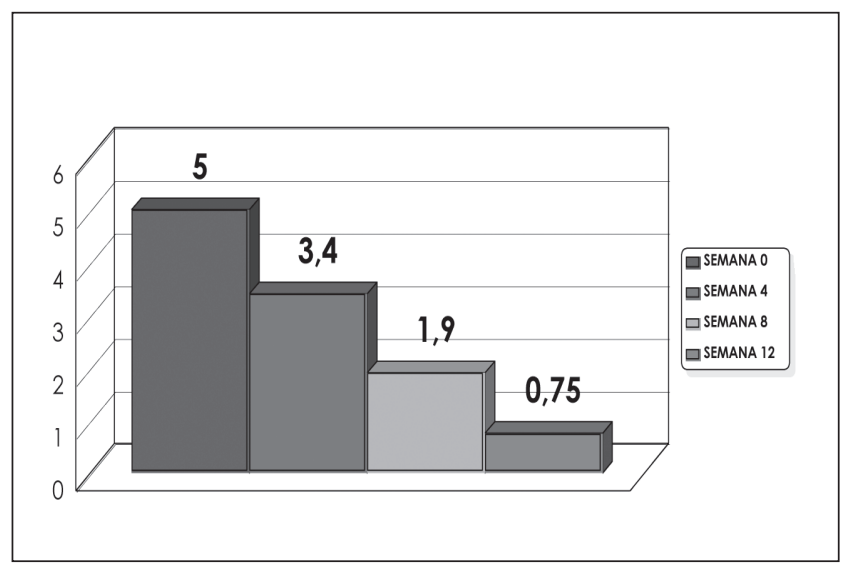

Figura 1. Resultados Escala Analógica Visual de Craving de Cocaína, p $<0.001$.

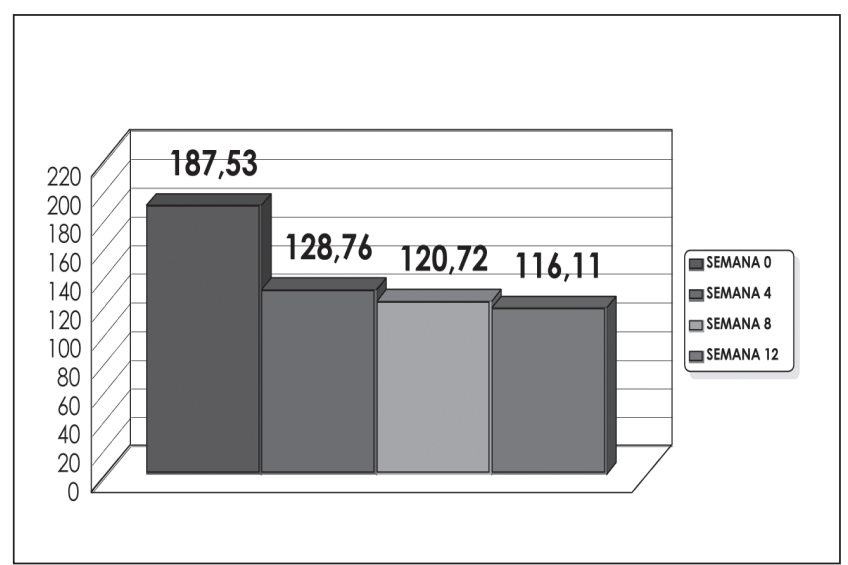

Figura 2. Puntuación Global de Craving (CCQ), p $<.0 .001$. 
Respecto a los Valores de Impulsividad (Figuras 3 a 6) se produce un descenso gradual en las puntuaciones hasta la semana 8 con un leve aumento en la medición a las 12 semanas. Este aumento en el tramo final del seguimiento se observa en impulsividad motora y no planificada pero en cambio sigue disminuyendo en impulsividad cognitiva. Pensamos que tal como va avanzando el tratamiento el control externo del paciente en tratamiento va siendo menor y esto también influye de diferente forma en los distintos componentes de la impulsividad. En este punto debemos destacar que aunque tomamos como referencia las puntuaciones obtenidas en población normal algunos autores proponen utilizar puntuaciones de referencia superiores a los de población general cuando se trata de una muestra de cocainómanos ${ }^{6,11}$.

La significación estadística para el contraste de puntuaciones t para muestras relacionadas es el siguiente: Impulsividad cognitiva Barratt ( $p<0.001)$ : descenso de 5,76 puntos; Impulsividad motora Barratt ( $p<0,001)$ : descenso de 9,15 puntos; Impulsividad no planeada Barratt $(p<0.001)$ : descenso de 7,73 puntos; Impulsividad total Barratt $(p<0.001)$ : descenso de 21,4 puntos.

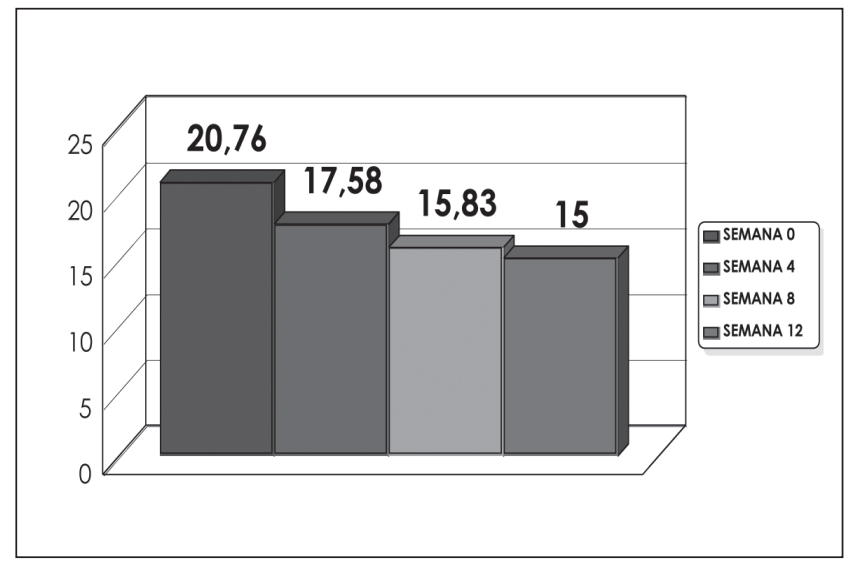

Figura 3. Impulsividad cognitiva de Barratt (Población Normal: 9,5), p $<0.001$.

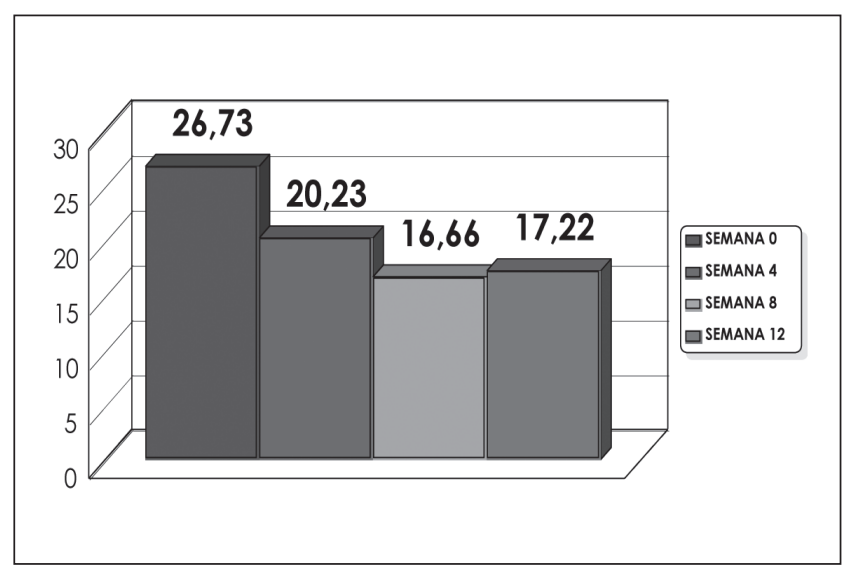

Figura 4. Impulsividad motora de Barratt (Población Normal: 9,5), p $<0.001$.

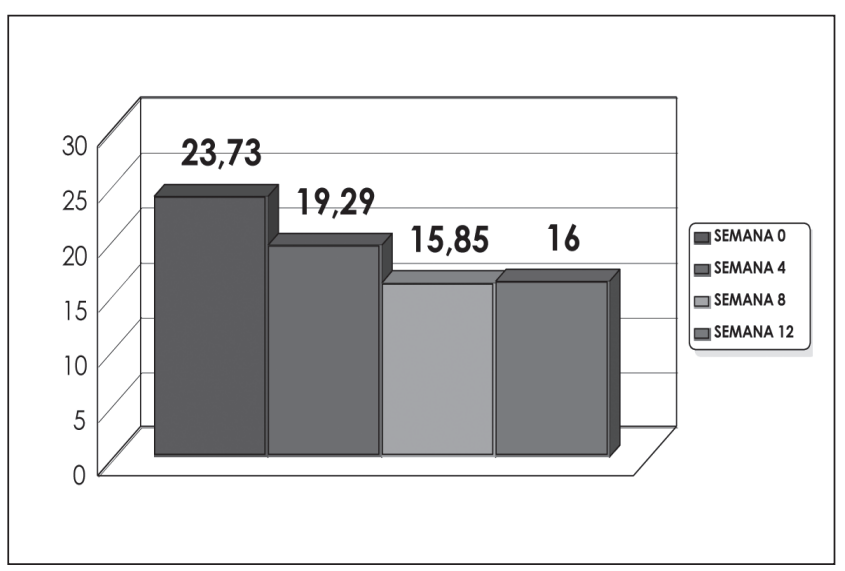

Figura 5. Impulsividad no planeada de Barratt (Población Normal: 14), p $<0.001$.

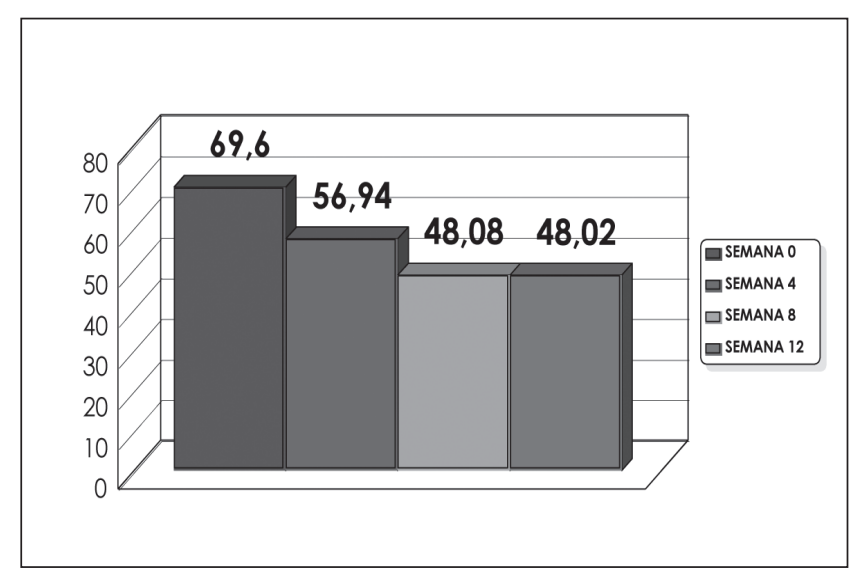

Figura 6. Impulsividad total de Barratt (Población Normal: 32,5$)$, p $<0.001$.

Durante el seguimiento de la evolución de los pacientes en tratamiento con Oxcarbacepina, tal y como ya hemos señalado previamente, tuvimos un importante porcentaje de pérdidas a lo largo del estudio, lo que ocasiona un sesgo que hemos tenido muy en cuenta y nos hace ser prudentes con nuestros resultados. La mayor parte de ellas se produjeron durante el primer mes, de forma que cerca de un $40 \%$ de la muestra abandono el seguimiento a la $4^{2}$ semana, este porcentaje descendió progresivamente al mismo tiempo que se incrementaba el índice de abstinencia, de forma que en el último mes, entre las 8 y las 12 semanas de tratamiento, tan solo abandonaron tres pacientes.

\section{Efectos secundarios.}

En cuanto a los Efectos Secundarios la intensidad de los síntomas fue muy leve ya que en ningún caso fue necesario reducir la dosis ni abandonar el tratamiento. La mayor parte de los síntomas remitieron a lo largo del tratamiento. Hay que señalar que un mismo 
paciente podía referir uno o más efectos secundarios. Destacan alteraciones cognitivas leves e incremento en el estado de ánimo (62\% en ambos casos). Este último síntoma más que un efecto secundario podría deberse a la evolución positiva del paciente. También hallamos un elevado porcentaje en los síntomas de adormecimiento $u$ hormigueos en los pies, 50\%.

\section{DISCUSIÓN}

Las características de consumo de cocaína y el perfil de la muestra de pacientes cocainómanos que participaron en el estudio son similares a los de gran parte de las publicaciones españolas revisadas sobre el mismo tema $6,11,21$.

La abstinencia de Cocaína a los tres meses en los pacientes en tratamiento obtenida en nuestro trabajo es ligeramente superior a la señalada en la mayoría de estudios $^{6,11,21,22}$. Los resultados son significativamente positivos con abstinencia del $100 \%$ de los pacientes que permanecen en tratamiento a las 12 semanas (partiendo del 30\% en la semana basal). La abstinencia de cocaína al primer mes es del $82 \%$ y del $90 \%$ de los pacientes al segundo mes de tratamiento con una $\mathrm{n}$ de 18 pacientes de los 30 que comenzaron el tratamiento.

El análisis de los datos del estudio, a pesar de las limitaciones del tamaño de la muestra y el alto porcentaje de abandonos al inicio del tratamiento ya anteriormente señalados, $40 \%$ en el primer mes, evidencia que el tratamiento con Oxcarbacepina apoya las hipótesis de partida ya que se logran descensos estadísticamente significativos del Craving y la impulsividad a las 4, 8 y 12 semanas con respecto a la medida basal al inicio del tratamiento.

Nuestros resultados de descenso estadísticamente significativo en los valores del craving a cocaína son ligeramente mayores a los hallados en otros estudios españoles con pacientes que abandonan el consumo de cocaína ${ }^{8,11,21,22}$

Un análisis de los resultados en la primera entrevista muestra que aquellos pacientes que abandonan el tratamiento ( $n=12$ ) obtenían al inicio del tratamiento unas puntuaciones superiores en impulsividad que aquellos que permanecen en él, exceptuando el apartado de impulsividad cognitiva, ya que a pesar de que existen puntuaciones superiores en casi 3 puntos la diferencia no tiene significación estadística.

En cuanto al craving encontramos un efecto muy interesante. En la Escala Analógica Visual de Craving, la muestra que abandona el tratamiento obtiene unas puntuaciones inferiores, mientras que en el caso del CCO se observa el efecto contrario.
La EAV de craving es de única respuesta y se trata de ubicarse en un continuo de 0 a 10 siendo evidente la lectura que el clínico hará de su respuesta. De hecho esta es una de las razones por la que cada vez están más en desuso y la mayor parte de los investigadores optan por otro tipo de medidas de mayor fiabilidad $^{8}, 14$. En el caso del CCO las preguntas están más elaboradas y no resulta tan obvia la interpretación de las respuestas. La negativa a admitir el verdadero grado de craving podría explicar en gran parte el abandono del tratamiento en aquellas personas cuya disposición esta más orientada a mantener el consumo que a conseguir la abstinencia.

La hipótesis podría ser que aquellas personas que abandonan el tratamiento mostraban en la primera entrevista una mayor deseabilidad social que aquellos que permanecen en él admitiendo un craving inferior al que realmente presentaban.

Esta hipótesis no sería descabellada si tenemos en cuenta que al principio del tratamiento es precisamente en los apartados de impulsividad motora y no planificada donde más puntúan los pacientes que abandonan y, en cambio, cognitivamente parecen mantener el menor nivel de impulsividad y por lo tanto conservan la capacidad para buscar la deseabilidad social inherente a todo entrevistado. Lamentablemente no podemos hacer un análisis más profundo de la muestra ya que no conocemos como evolucionan aquellos pacientes que abandonan el tratamiento en relación al craving e impulsividad.

Los valores de impulsividad, en nuestro caso, y a pesar del descenso significativo en las puntuaciones, no se llegan a equiparar a los valores de la población normal. En cualquier caso existe bibliografía abundante $^{11,13,14,15,23}$ acerca de la comorbilidad del consumo de cocaína con trastornos del control de impulsos por lo que es muy posible que si tomásemos mediciones en momentos más avanzados del tratamiento tampoco se alcanzasen los valores obtenidos en población normal. Algunos autores proponen utilizar, para el Barratt, puntuaciones de referencia superiores a los de población general cuando se trata de evaluar la impulsividad en muestras de cocainómanos ${ }^{6}$. 11. Parecen ser factores decisivos en la retención al tratamiento en cocainómanos los niveles de impulsividad al inicio del tratamiento. De forma que a menor impulsividad cognitiva y mayor impulsividad motora y no planificada menor retención.

Las perdidas a lo largo del seguimiento en nuestro estudio, 12 pacientes, a pesar de representar la mayor limitación para extraer unas conclusiones categóricas son muy similares a los resultados de adherencia a tratamiento en pacientes cocainómanos en nuestro entorno, entre un 40-60 \% de abandonos $3,21,22$ 
Como se ha señalado en una reciente publicación que revisa ampliamente los estudios sobre tratamientos en pacientes con trastorno por abuso / dependencia a cocaína ${ }^{8}$, y tal como reiteradamente indican los trabajos de seguimiento clínico en consumidores de drogas ${ }^{11,12,21}$, las perdidas en este tipo de estudios suelen ser elevadas sobre todo en las primeras semanas, las causas son muy diversas y no siempre totalmente atribuibles al paciente, nosotros pensamos que muchas veces la respuesta inicial de la estructura terapéutica a la demanda es determinante para la continuidad en el tratamiento.

Los datos deben ser tomados con cautela dado que la adherencia al tratamiento ha sido muy baja con perdidas cercanas al $40 \%$ en el primer mes de tratamiento, aunque en esta línea debemos apuntar resultados que reflejan la homogeneidad de esta problemática con pacientes cocainómanos en las que las tasas de permanencia en las redes de asistencia pública no superan el $40 \% 8$.

\section{CONCLUSIONES}

La oxcarbacepina podría ser eficaz en la reducción de la impulsividad y también sobre el craving de cocaína.

Al mismo tiempo el tratamiento con oxcarbacepina parece mejorar la evolución de los pacientes cocainómanos que se mantienen en tratamiento con una abstinencia del $100 \%$ a los tres meses $160 \%$ de los que inician el tratamiento).

Los pacientes que al inicio del tratamiento presentan elevados niveles de impulsividad motora y no planificada y menores puntuaciones en impulsividad cognitiva tienen mayores tasas de abandono.

En nuestra experiencia pensamos que dosis de oxcarbacepina superiores a los 900 mgr. y posiblemente iniciando con dosis más elevadas desde el primer día de tratamiento, puede mejorar la retención de los pacientes especialmente en aquellos que presentan los niveles más elevados de impulsividad motora y no planificada, y más teniendo en cuenta la mínima incidencia de efectos secundarios relevantes acontecidos a lo largo del presente estudio.

Recomendamos realizar estudios de seguimiento que analicen los valores de impulsividad en aquellos pacientes que abandonan tratamiento.

Conflicto de intereses: Los autores declaran que el presente trabajo no ha recibido financiación económica ni apoyo de ninguna entidad publica o privada, ni de la industria farmacéutica.

\section{REFERENCIAS}

1. OEDT. El problema de la Drogodependencia en España. Informe anual 2005. EMMCDA. Lisboa. 2005

2. DGPNSD. Encuesta Estatal sobre Uso de Drogas en Enseñanzas Secundarias (ESTUDES). Ministerio Sanidad i Consumo. 2004. Madrid. Observatorio Español Sobre Drogas. Situación y tendencias de los problemas de drogas en España. Informe 2004. DGPNSD. Ministerio Sanidad y Consumo. Madrid 2005.

3. GMD. Ayuntamiento de Castellón. Informe SECAD Generalitat Valenciana, Consellería de Sanitat. Memoria Proyecto Amigo 2005 y Memoria asistencial 2005 UCA Castelló. Consellería de Sanitat. Informes no publicados.

4. Pastor R, Llopis JJ, Baquero A. Interacciones y consecuencias del consumo combinado de alcohol y cocaína: Una actualización sobre el cocaetileno._Adicciones 2003; 15: 159-165.

5. Nestler EJ. The neurobiology of cocaine addiction. Science Practice and Perspectives. NIDA 2005; 3, 1 : 4-11.

6. Prieto J, Álvarez A, Olea M, Montero I. Utilización de la Gabapentina durante la desintoxicación de pacientes cocainómanos. Trastornos Adictivos 2001; 3: 287-288

7. Salivas P, McFarland K, Bowers M, Szumlinski K, Xi Z, Baker D. Glutamate Transmission and Addiction to cocaine. En: Moghaddan, B. Wolf,M. (eds.): Glutamate and disorders of cognition and motivation. Ann. N.Y. Acad. Sci 2003; 1003: 169-175

8. López Duran A, Becoña E. El craving en personas dependientes de cocaína. Anales de Psicología 2006; 22: 205-211.

9. Llopis, J.J. Dependencia, intoxicación y síndrome de abstinencia por Cocaína. En: Pascual P, Torres M, Calafat A. Monografía Cocaína. Adicciones. 2001; 13, Supl. 2: 147-166.

10. Caballero Martínez L. Adicción a la cocaína: neurobiología, clínica, diagnóstico y tratamiento. Delegación del Gobierno para el Plan sobre Drogas. Ministerio Sanidad y Consumo. Madrid. 2005.

11. Sáez C, Camáñes C, García M, Torres M, García JM, Martínez L. Eficacia de Gabapentina y oxcarbacepina en el control de la ansiedad e impulsividad de pacientes con abuso/ dependencia de cocaína o cocaína y alcohol. Actas Esp. Psiquiatr 2003; 31 (supl. 1); 126.

12. Kamppman K, Pettinati H, Lynch K, Dackis C, Sparkman T, Weigley C, O'Brien P. Ensayo piloto de topiramato en el tratamiento de la dependencia de cocaína. Drug Alcoh Depend 2004; 75: 230-240.

13. Conejo, J. Laita, P. Moñino, L. Eficacia antiimpulsiva de oxcarbacepina en los trastornos de personalidad. Psiq Biol. 2005; 12: 95- 100.

14. Mena A, Döll A, Gairibi A. Utilidad de la oxcarbacepina en la desintoxicación de pacientes politoxicómanos. Adicciones. Suplemento XXXIII Jornadas Nacionales de Socidrogalcohol 2006. 180. 
15. Ait-Daoud N, Malcom R, Johnson B. An overview of medications for the treatment of alcohol withdrawal and alcohol dependence with an emphasis on the use of older and newer anticonvulsants. Addict Behav. 2006 Sep; 31: 1628-49

16. Tiffany, S. Singlenton, E. Haertzen, C. y Henmingfield, I. The development of a cocaine craving cuestionaire. Drug Alcoh Depend 1993; 34: 18-28.

17. Tejero A, Trujols J. Siñol Ñ. Evaluación del craving de cocaína. En: Pérez de los Cobos, J. Tejero, A. Trujols, J.: Instrumentos clínicos para la evaluación de la dependencia a cocaína. 2003 Psiquiatría Editores. Ed. Ars. Médica. Barcelona. 23-50.

18. Magenta P, Arghetti S, Di Palma F, Jann S, Sterlicchio M, Bianconi C, Galimberti V, Oslo M, Siciliano G, Cavalloti G, Sterzi R. Oxcarbazepine is effective and safe in the treatment of neuropathic pain: pooled analysis of seven clinical studies. Neurol Sci 2005; 26: 218-226.

19. Oquendo M, Baca-García E, Graver R, Morales V, Mann J. Spanish adaptation of the Barratt impulsiveness scale, (Bis-11). Eur J Psychiatry 2001; 15: 147-155.
20. García Portilla MP, Bobes J, Bascaran MT, Saiz P, Bousoño M. Banco de instrumentos básicos para la práctica de la psiquiatría clínica. $4^{a}$ edición. Ed. Ars Médica. Barcelona. 2006.

21. López Duran A, Becoña Iglesias E, García Janeiro J, Senra Comesaña A, Cancelo Martinez J, Estévez Vorkauf C, Sobradejo Lens J, Viete Fernández I, Lloves Moratinos M, Moneo Montes A, Casete Fernández L, Lage López M, Diaz Castro E. ¿Como evolucionan las personas con dependencia de la cocaína que están en tratamiento? Estudio a los 3 y 6 meses. Adicciones 2006; 18: 327-335.

22. Forcada R, Pardo N, Bondia B. Impulsividad en dependientes de cocaína que abandonan el consumo. Adicciones 2006; 18: 111-118.

23. Almoguera J, Henche A, Díaz A. El amisulpiride como tratamiento de los síntomas psicóticos en pacientes dependientes de cocaína. XXXIII Jornadas Nacionales de Socidrogalcohol 2006. 94-95. 